OPEN ACCESS

Edited by:

Sarit Ashkenazi,

The Hebrew University of Jerusalem,

Israel

Reviewed by:

Patrizia Silvia Bisiacchi,

University of Padova, Italy

Fuhong $L i$,

Jiangxi Normal University, China

*Correspondence:

Xinlin Zhou

zhou_xinlin@bnu.edu.cn

Specialty section:

This article was submitted to

Cognition,

a section of the journal

Frontiers in Psychology

Received: 02 December 2015

Accepted: 17 February 2016

Published: 07 March 2016

Citation:

Wei W, Chen C and Zhou X (2016) Spatial Ability Explains the Male Advantage in Approximate Arithmetic.

Front. Psychol. 7:306.

doi: 10.3389/fpsyg.2016.00306

\section{Spatial Ability Explains the Male Advantage in Approximate Arithmetic}

\author{
Wei Wei,2, Chuansheng Chen ${ }^{3}$ and Xinlin Zhou ${ }^{2 *}$ \\ ${ }^{1}$ Department of Psychology and Behavioral Sciences, Zhejiang University, Hangzhou, China, ${ }^{2}$ State Key Laboratory of \\ Cognitive Neuroscience and Learning, Siegler Center for Innovative Learning, Beijing Normal University, Beijing, China, \\ ${ }^{3}$ Department of Psychology and Social Behavior, University of California, Irvine, CA, USA
}

Previous research has shown that females consistently outperform males in exact arithmetic, perhaps due to the former's advantage in language processing. Much less is known about gender difference in approximate arithmetic. Given that approximate arithmetic is closely associated with visuospatial processing, which shows a male advantage we hypothesized that males would perform better than females in approximate arithmetic. In two experiments (496 children in Experiment 1 and 554 college students in Experiment 2), we found that males showed better performance in approximate arithmetic, which was accounted for by gender differences in spatial ability.

Keywords: gender difference, approximate arithmetic, spatial ability

\section{INTRODUCTION}

Gender differences in mathematical performance have been an important area of research because researchers and policy makers alike have been concerned about the under representation of women in mathematics-intensive fields or Science, Technology, Engineer, and Mathematics (STEM; Hyde and Linn, 2006; Halpern et al., 2007; Guiso et al., 2008; Hyde et al., 2008; Ceci et al., 2009; Nosek et al., 2009; Else-Quest et al., 2010; Shen, 2013). Many studies have been conducted to investigate the cognitive, socio-cultural, and biological origins of these differences (Halpern et al., 2007; Kovas et al., 2007; Guiso et al., 2008).

Although male advantage in mathematics has been widely reported, it is by no means the only story in town (Spelke, 2005). For example, Hyde and Linn (2006), Hyde et al. (2008) have emphasized the gender similarity hypothesis. Moreover, there is evidence that at an early age females show better performance in arithmetic than do males (Linn and Hyde, 1989; Wei et al., 2012). One possible explanation of such an advantage is that arithmetic tends to rely on language processing (Dehaene et al., 1999; Lemer et al., 2003), which shows a female advantage (Wei et al., 2012). As Wei et al. (2012) found, after controlling for verbal ability, gender differences in mathematical performance disappeared.

Some arithmetic tasks, however, may not involve much language processing. Distinct from exact arithmetic, approximate arithmetic (e.g., "Of 3 and 8, which number is closer to the answer to the problem $4+5$ ?") is believed to involve less verbal processing but more number sense and visuospatial processing (Dehaene et al., 1999). Studies have found that approximate arithmetic could be performed without symbols and language (Pica et al., 2004). Young children without formal education can perform large-number symbolic approximate arithmetic (Gilmore et al., 2007). Neuroimaging studies further supported the distinction between exact and approximate arithmetic. It has been found that exact arithmetic relies on the language system, whereas 
approximate arithmetic relies on the numerical magnitude processing system or the internal "number line" (Dehaene et al., 1999). Specifically, approximate arithmetic recruits the parietal lobe, which is involved in visuo-spatial processing.

In the current study, we recruited two age groups of students to examine gender differences in approximate arithmetic. Given that males show better performance in spatial ability (Voyer et al., 1995) and that spatial ability is linked to approximate arithmetic as mentioned above, we hypothesized that males would outperform females in approximate arithmetic, and that spatial ability would be the cognitive mechanism for the gender difference in approximate arithmetic.

\section{EXPERIMENT 1}

\section{Materials and Methods Participants}

Children in 6th-8th grades students were recruited for the study. Children came from two Chinese cities, Liuzhou (Guangxi Province) and Beijing. There were 496 children (234 males and 262 females), 11.0-15.9 years old. All participants were native Chinese speakers and had normal or corrected-to-normal eyesight. This study was approved by the Institute of Cognitive Neuroscience and Learning at Beijing Normal University and the principals of the schools.

\section{Procedure}

Participants took computerized mathematical and other cognitive tests in a computer room in groups of about 30-40 students per class. They were monitored by 2-3 experimenters and, in the case of 6th-8th grade students, by the class's teacher as well. Instructions and a practice session were given before each formal test. The tasks were administered in the same order for all students. Participants responded by pressing "P" or "Q" on the keyboard for three of the five tasks (see below), using the mouse for the spatial working memory task, and entering a numerical value for the approximate arithmetic task. Participants' responses were automatically recorded and sent over the internet to a server located in our laboratory at the university.

\section{Tasks}

All the tasks were programmed using Web-based applications available at: www.dweipsy.com/lattice (Wei et al., 2012).

\section{Symbolic approximate arithmetic}

This task was based on Levine's (1982) Test of Estimation Ability (TEA). The open-ended paradigm (Levine, 1982; Rubenstein, 1985; Dowker, 1992; Dowker et al., 1996) was adopted in the current study to test the ability of approximate arithmetic. An equation was presented in the middle of the screen. On the top the screen, there was a time bar, indicating $15 \mathrm{~s}$. To ensure that the participants could not calculate the exact answer in 15 s, we used multiple digits for all equations (see Table 1). Participants were asked to come up with the best approximate answer for the equation in $15 \mathrm{~s}$. Participants entered the answer into an input box at the bottom of the screen. The formal test included 40 trials, including addition, subtraction, multiplication, and division. The four operations were presented randomly for each participant. Both integral and decimal arithmetic was used in this task.

\section{Three-dimensional mental rotation}

This task was based on Shepard's mental rotation task (Shepard and Metzler, 1971). For each trial, one three-dimensional image was presented on the upper part of the screen, and two others on the lower part. Participants were asked to choose one from the bottom to match with the top; the matching image could be identified only by mental rotation. Participants were asked to press the "Q" key if he/she chose the image on the left, or the "P" key he/she chose the image on the right. The formal test included 180 trials and was limited to $3 \mathrm{~min}$. The rotation angles of the images were $15^{\circ}, 30^{\circ}, \ldots, 345^{\circ}$, with a step of $15^{\circ}$. Each trial would remain on the screen until participants responded by pressing "P” or “Q”.

\section{Raven's progressive matrices}

The Raven's Progressive Matrices test (Raven, 1998) was used to assess general intelligence. In this test, participants needed to identify the missing segment of a figure according to the figure's inherent regularity. They should press "Q" if the missing segment was on the left or "P" if it appeared on the right. The formal test included 80 trials and was limited to $4 \mathrm{~min}$.

\section{TABLE 1 | The test of symbolic approximate arithmetic.}

\begin{tabular}{|c|c|c|c|c|}
\hline Item $\quad$ Operation & Addition & Subtraction & Multiplication & Division \\
\hline 1 & $1752+9339$ & $8473-1247$ & $581 \times 64$ & $6.664 \div 0.98$ \\
\hline 2 & $8928+5397$ & $10395-13657$ & $735 \times 44$ & $4144 \div 37$ \\
\hline 3 & $4578+3566$ & $27534-11846$ & $23 \times 76$ & $23596 \div 68$ \\
\hline 4 & $8546+5773$ & $7814-1937$ & $397 \times 35$ & $11515 \div 47$ \\
\hline 5 & $3696+1276$ & $57631-14768$ & $34 \times 87$ & $16068 \div 78$ \\
\hline 8 & $6.759+0.2867$ & $5.614-10.4935$ & $0.893 \times 3.7$ & $403.76 \div 0.98$ \\
\hline 9 & $926.4+75.72$ & $208.3-129.26$ & $2.17 \times 0.83$ & $66.3 \div 6.5$ \\
\hline 10 & $38.69+629.8$ & $15.94-10.798$ & $0.68 \times 7.9$ & $343.2 \div 22$ \\
\hline
\end{tabular}




\section{Spatial working memory}

This task was similar to Corsi block task (Corsi, unpublished doctoral dissertation). Non-overlapping dots were sequentially presented in an implicit lattice of $3 \times 3$ on the computer screen. Each dot was presented for $1 \mathrm{~s}$, and dots were presented with an interval of $1 \mathrm{~s}$. After the last dot was presented and disappeared, a cue would be presented on the screen to ask the participants to click the positions where the dots had appeared in the same sequence as their appearance. The number of dots ranged from 3 to 7. There was no feedback to participants. The average distance between the position where the dot appeared and the position where participants clicked was calculated and treated as an index of spatial working memory.

\section{Word semantic processing}

The format of this task was similar to the one used by Siegel and Ryan (1988) and So and Siegel (1997). Materials in the task were adapted from the language examinations used in China in recent years. In the task, a sentence was presented in the center of the computer screen with a word missing. Participants needed to select one of two candidate words presented beneath the sentence by pressing a left or a right key. The stimulus remained on the screen until the participants responded. The formal test included 120 trials and was limited to $5 \mathrm{~min}$.

For each of the time-limited tasks (i.e., mental rotation, Raven's Progressive Matrices, and word semantic processing), we calculated scores using Guilford formula (Guilford proposed a correction formula " $S=R-W /(n-1)$ " (S: the adjusted number of items that the participants can actually perform without the aid of chance. R: the number of correct responses, W: the number of incorrect responses. $n$ : the number of alternative responses to each item; Guilford, 1936). For the spatial working memory task, as mentioned earlier, the average distance between the position where the dot appeared and the position where participants clicked was calculated. We then subtracted the average distance from 200 to create a score for spatial working memory. For the approximate arithmetic task, we used the formula "100 - |(PR $\mathrm{EA}) /(\mathrm{PR}+\mathrm{EA}) \mid \times 100$ " to calculate accuracy in approximate arithmetic. PR refers to participant's response and EA the exact answer. Using this formula, accuracy scores in approximate arithmetic would have the theoretical range from 0 to 100.

\section{Data Analysis}

Because our sample came from 20 classes, it was necessary to first investigate whether the nested data needed to be analyzed with multilevel models. We used the unconditional means model to compute the intraclass correlation coefficients (ICC) (Peugh and Enders, 2005). The ICC was 0.12 for approximate arithmetic, suggesting significant variability at the between-classroom level. Therefore, we conducted multilevel models by using the MIXED procedure in SPSS for all data analyses. The following equations were used:

$$
\begin{aligned}
\text { Level } 1: \text { Score }_{i j}= & \beta_{0 j}+\beta_{1 j}\left(\text { Age }_{i j}\right)+\beta_{2 j}\left(\text { Gender }_{i j}\right)+ \\
& \beta_{3 j}\left(\text { Covariates }_{i j}\right)+\gamma_{i j}
\end{aligned}
$$

Where Score $_{i j}$ was the score of approximate arithmetic for participant $i$ in class $j$, and $\beta_{0 j}$ was the mean score for class $j . \beta_{1 j}$, $\beta_{2 j}$, and $\beta_{3 j}$ were the slopes of age, gender and covariates (i.e., scores of various tests) predicting the score within class $j . \gamma_{i j}$ was the random component of the score for participant $i$ in class $j$.

$$
\begin{aligned}
\text { Level 2: } & \beta_{0 j}=\gamma_{00}+\gamma_{01} \text { Region }_{i j}+\mu_{0 j} \\
\beta_{1 j} & =\gamma_{10} \\
\beta_{2 j} & =\gamma_{20} \\
\beta_{3 j} & =\gamma_{30}
\end{aligned}
$$

Where $\beta_{0 j}$ was the mean score for class $j, \gamma_{00}$ was the grand mean score across all classes, $\gamma_{01}$ was the slope of level- 2 variable region predicting the mean score for class $j$, and $\mu_{0 j}$ was the random component of the mean score for class $j . \beta_{1 j}, \beta_{2 j}$, and $\beta_{3 j}$, were the slopes of age, gender and covariates predicting the mean score for class $j$.

Combined:

$$
\begin{aligned}
\text { Score }_{i j}=\gamma_{00} & +\gamma_{01}(\text { Region }) \\
& +\gamma_{10}(\text { Age }) \\
& +\gamma_{20}(\text { Gender })+\gamma_{30}(\text { Covariates })+\mu_{0 j}+\gamma_{i j}
\end{aligned}
$$

\section{Results and discussion}

Of the 19840 answers (496 children $\times 40$ trials), $210(1.1 \%)$ were correct exact answers. Table 2 shows the mean scores and standard deviations of all tasks. Table 3 shows the inter-task correlations. All correlations were significant.

According to multilevel model analysis, boys outperformed girls in approximate arithmetic and mental rotation, whereas girls outperformed boys in word semantic processing and Raven's Progressive Matrices. There was no gender difference in spatial working memory (Table 2).

The analysis showed no differences between older and younger children for all tasks $[b=-1.13, t(330)=-$ $0.54, p=0.586$ for symbolic approximate arithmetic; $b=0.57, t(168)=0.60, p=0.551$ for mental rotation; $b=-0.94, t(222)=-1.21, p=0.227$ for word semantic processing; $b=1.17, t(249)=0.47, p=0.638$ for spatial working memory; $b=0.95, t(216)=1.51, p=0.133$ for Raven's Progressive Matrices]. In the multilevel model (when classroom effect was considered), no region differences were found for all tasks $[b=-5.49, t(18)=-1.61, p=0.125$ for symbolic approximate arithmetic; $b=0.96, t(16)=0.81, p=0.429$ for mental rotation; $b=-1.42, t(21)=-1.41, p=0.172$ for word semantic processing; $b=-4.73, t(20)=-1.38, p=0.181$ for spatial working memory; $b=1.10, t(15)=1.25, p=0.229$ for Raven's Progressive Matrices]. None of the interactions involving gender and approximate arithmetic were significant $[b=0.12, t(477)=0.03, p=0.979$ for gender $\times$ region; $b=5.26, t(479)=1.06, p=0.288$ for gender $\times$ age; $b=-5.37$, $t(476)=-0.079, p=0.431$ for gender $\times$ region $\times$ age $].$

Multilevel model analysis showed that after controlling for mental rotation, gender difference in approximate arithmetic disappeared (Table 4 and Figure 1). After controlling for any one of the other measures, however, gender difference 


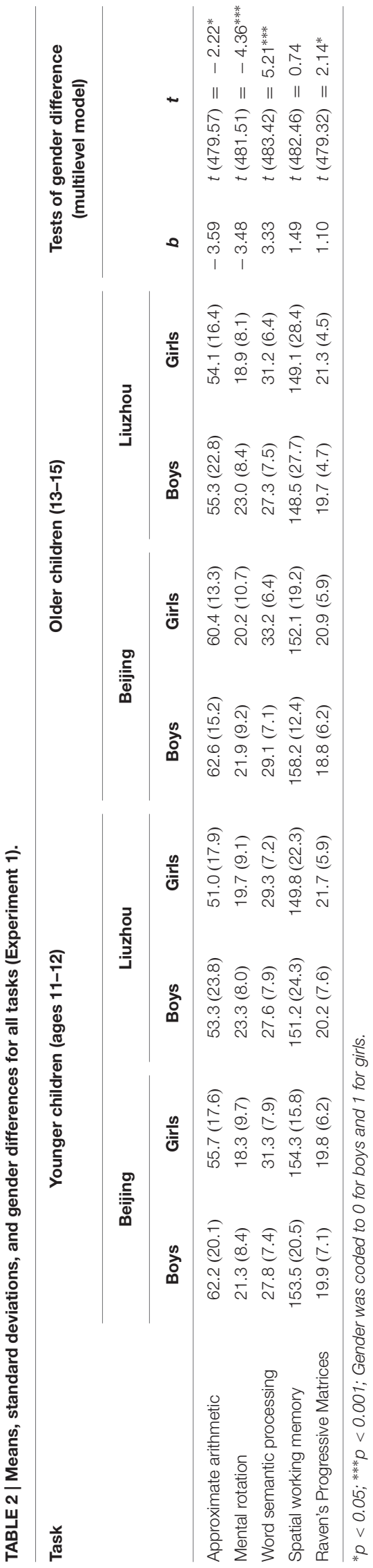

TABLE 3 | Correlations among all tasks (Experiment 1).

\begin{tabular}{|c|c|c|c|c|c|}
\hline \multicolumn{2}{|c|}{ Tasks } & \multirow{2}{*}{$\begin{array}{l}1 \\
-\end{array}$} & \multirow[t]{2}{*}{2} & \multirow[t]{2}{*}{3} & \multirow[t]{2}{*}{4} \\
\hline 1 & Approximate arithmetic & & & & \\
\hline 2 & Mental rotation & $0.16^{* *}$ & - & & \\
\hline 3 & Word semantic processing & $0.21^{* * *}$ & $0.12^{* *}$ & - & \\
\hline 4 & Spatial working memory & $0.31^{* * *}$ & $0.20^{* * *}$ & $0.16^{* * *}$ & - \\
\hline 5 & Raven's Progressive Matrices & $0.14^{* *}$ & $0.20^{* * *}$ & $0.12^{* *}$ & $0.13^{* *}$ \\
\hline
\end{tabular}

${ }^{* *} p<0.01 ;{ }^{* * *} p<0.001$.

TABLE 4 | Results from multilevel modeling showing gender differences in approximate arithmetic (Experiment 1).

\begin{tabular}{lccc}
\hline Covariate & \multicolumn{3}{c}{ Approximate arithmetic } \\
\cline { 2 - 4 } & $\boldsymbol{b}$ & SE $\times \boldsymbol{b}$ & $\boldsymbol{t}$ \\
\hline None & -3.59 & 1.61 & $t(479.57)=-2.22^{*}$ \\
Mental rotation & -2.73 & 1.64 & $t(481.02)=-1.66$ \\
Word semantic processing & -5.02 & 1.64 & $t(477.95)=-3.06^{*}$ \\
Spatial working memory & -3.21 & 1.57 & $t(479.47)=-2.05^{*}$ \\
Raven's Progressive Matrices & -4.02 & 1.61 & $t(478.10)=-2.50^{*}$
\end{tabular}

${ }^{*} p<0.05 ;{ }^{* *} p<0.01$; Gender was coded as 0 for boys and 1 for girls. SE: standard error.

in approximate arithmetic still remained (Table 4). Even after controlling for all the other measures simultaneously, gender difference in approximate arithmetic remained (Figure 1), $b=-$ $4.74, t(476.27)=-2.97, p=0.003$.

We further examined whether mental rotation could explain the gender differences in other tasks. The results showed that these gender differences could not be explained by mental rotation: including gender difference in word semantic processing, $b=3.76, t(485.73)=5.82, p<0.0001$; and in Raven's Progressive Matrices: $b=1.57, t(482.95)=3.05, p=0.002$.

To further examine whether gender differences were consistent across the four arithmetic operations (i.e., addition, subtraction, multiplication, and division), we re-conducted the multilevel model analysis, with arithmetic operation as a within-subject variable and gender, age, and region as betweensubject variables. Results showed that gender, age, and region had significant main effects, $b=-2.80, t(1883)=-2.62, p=0.009$ for gender, $b=2.70, t(1883)=2.41, p=0.016$ for age, and $b=6.65, t(1883)=6.03, p<0.001$ for region. No significant interaction effects were found among the variables, $b=0.11$, $t(1883)=0.10, p=0.920$. That is, boys outperformed girls for each operation (Figure 2). Controlling for scores on the mental rotation task, gender difference in approximate arithmetic was no longer significant, $b=-1.71, t(1871)=-1.59, p=0.113$.

The current investigation focused on children from primary and secondary schools in two regions of China. As expected, boys performed better than girls on approximate arithmetic. When we controlled for the three-dimensional mental rotation task, gender difference in approximate arithmetic disappeared. However, after controlling for the other cognitive tasks, gender difference in approximate arithmetic remained.

To our knowledge, little research has been conducted to explore the development of gender difference in arithmetic. Thus, the second experiment was conducted to investigate whether 


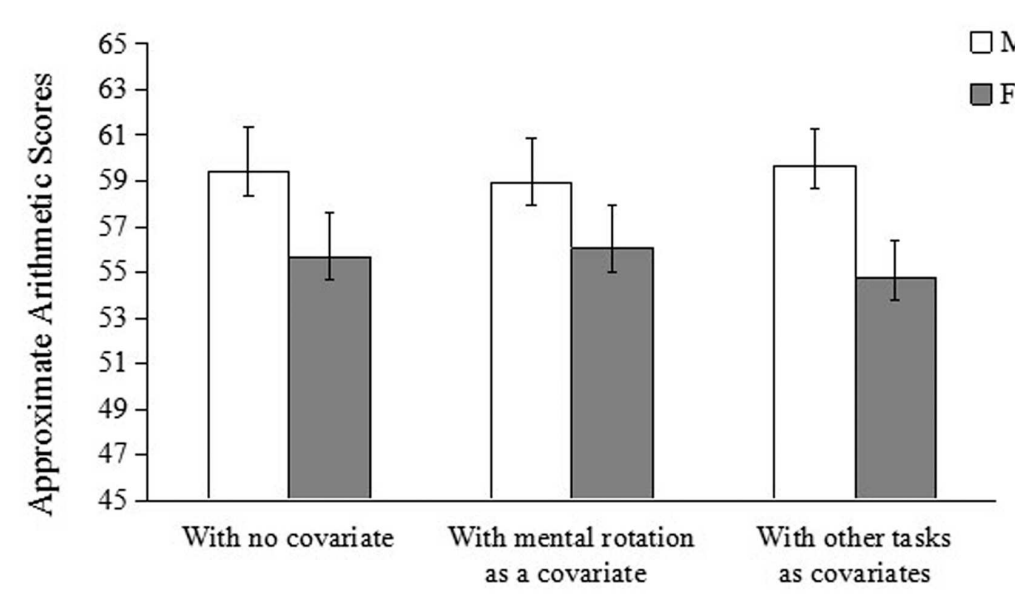

Children

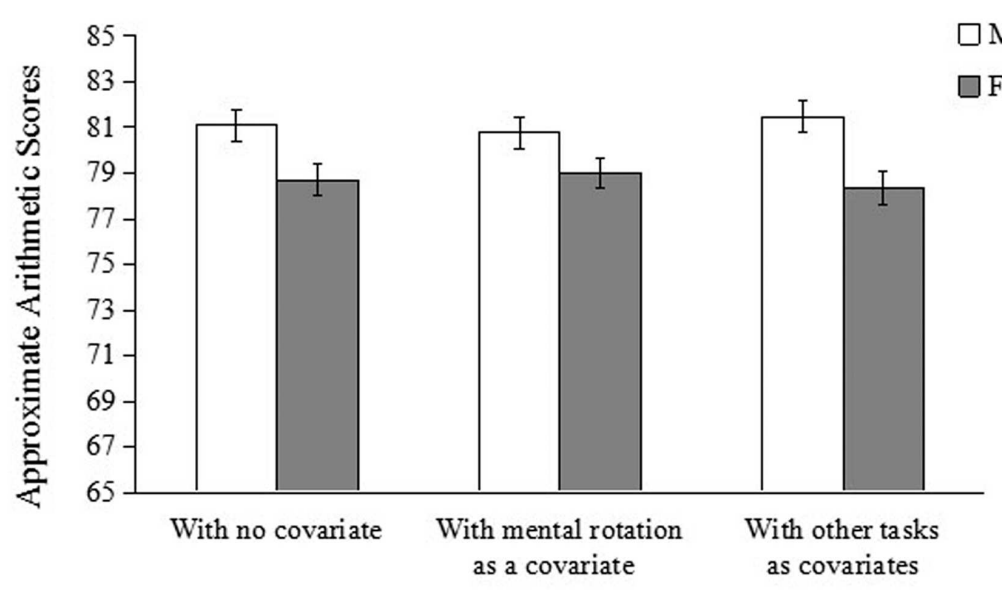

Adults

FIGURE 1 | Average scores in approximate arithmetic of children in Experiment 1 (top) and adults in Experiment 2 (bottom). The bars on the left show the means without controlling for covariate; the bars in the middle show the adjusted means after controlling for performance on the mental rotation task only; and the bars on the right show the adjusted means after controlling for performance on all other tasks except mental rotation task. Error bars indicate standard errors.

the gender differences in approximate arithmetic would exist in adults, and whether the same cognitive mechanisms would explain such gender differences.

\section{EXPERIMENT 2}

\section{Materials and Methods \\ Participants}

The adult sample of 554 college students (250 males and 304 females, 18.0-21.9 years old) was recruited from Harbin Normal University and Southwest University. It included 292 students majoring in sciences such as chemistry, computer science, biology, mathematics, and physics, and the others majoring in arts and humanities such as Chinese literature, education, history, and political science. All participants were native Chinese speakers and had normal or corrected-to-normal eyesight. They gave written consent form after procedure was fully explained. They received $30 \mathrm{RMB}$ (about US\$ 4.8) as a compensation for their time.

\section{Procedure and Tasks}

The procedure and tasks were the same as in Experiment 1.

\section{Data Analysis}

Similar multilevel models as in Experiment 1 were used in the current data analysis. The main equation was as follows:

$$
\begin{aligned}
\text { Score }_{i j}=\gamma_{00} & +\gamma_{01}(\text { Major })+\gamma_{10}(\text { Gender }) \\
& +\gamma_{20}(\text { Covariates })+\mu_{0 j}+\gamma_{i j}
\end{aligned}
$$

\section{Results and Discussion}

Ten participants (seven males and three females) were deleted as outliers because their approximate arithmetic scores were 3 


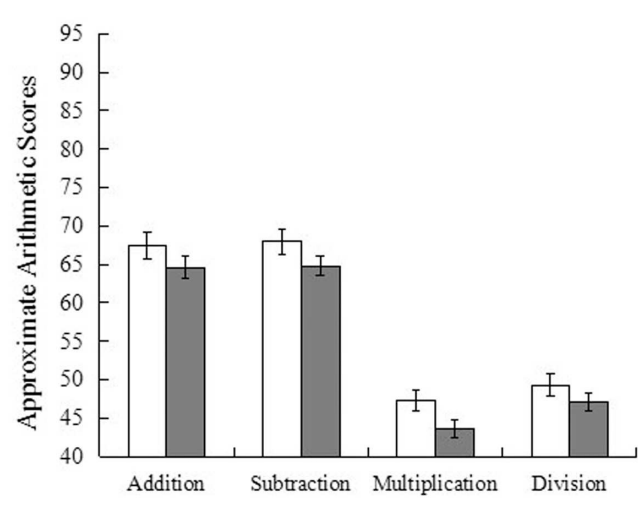

Children

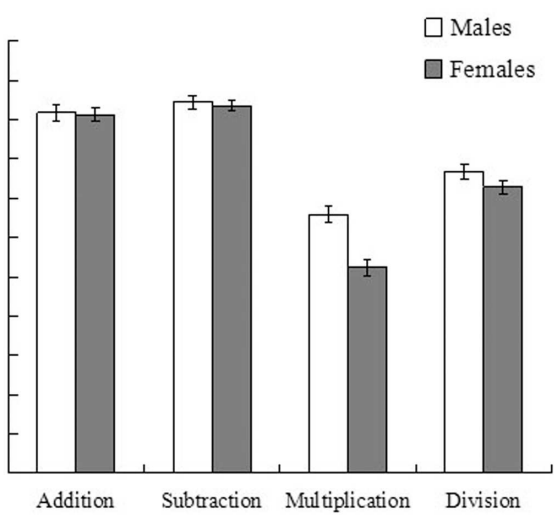

Adults

FIGURE 2 | Mean scores of approximate arithmetic (addition, subtraction, multiplication, and division) of children in Experiment 1 (left) and adults in Experiment 2 (right). Error bars indicate standard errors.

SD above or below the group mean. Of the remaining 21760 responses (544 participants $\times 40$ trials), 1481 responses $(6.8 \%)$ were exact answers. Table 5 shows the mean scores and standard deviations of all tasks. Table $\mathbf{6}$ shows the inter-task correlations. All correlations were significant.

Males outperformed females in approximate arithmetic and mental rotation, whereas females outperformed males in word semantic processing. There was no gender difference in spatial working memory and Raven's Progressive Matrices. Science students were superior to arts students in approximate arithmetic, $b=2.43, t(551)=2.55, p=0.011$. No difference across majors was found for other tasks: Raven's Progressive Matrices, $b=0.52, t(6.48)=0.59, p=0.577$; spatial working memory, $b=2.40, t(4.51)=1.21, p=0.286$; word semantic processing, $b=-0.86, t(6.36)=-0.63, p=0.550$. The interaction between gender and major was not significant $[b=-0.70$, $t(550)=-0.37, p=0.715]$.

Results showed that after controlling for mental rotation, gender difference in approximate arithmetic disappeared (Table 7 and Figure 1). But after controlling for other tasks, gender difference in approximate arithmetic remained (Table 7 and Figure 1). We further examined whether mental rotation could explain gender differences in performance on other tasks. The results showed that gender differences in word semantic processing could not be explained by mental rotation, $b=-3.32$, $t(549.87)=-5.62, p<0.001$.

To examine whether other cognitive tasks except for mental rotation could explain gender difference in approximate arithmetic, we controlled for spatial working memory, word semantic processing, and Raven' Progressive Matrices simultaneously. Gender difference remained, $b=2.81$, $t(548)=2.98, p=0.003$.

To further examine whether gender differences were consistent across the four arithmetic operations (i.e., addition, subtraction, multiplication, and division), we conducted repeated measure ANOVA with gender and students' major as between-subject variables. Results showed that gender $[b=3.01$, $t(2006)=2.19, p=0.029]$ and major $[b=4.02, t(2006)=2.86$, $p=0.004$ ] had significant main effects but no significant interaction $[b=0.72, t(2006)=1.15, p=0.250]$. Males outperformed females, and science students outperformed arts and humanities students on each operation. Results showed that after controlling for scores on the mental rotation task, gender differences in approximate arithmetic disappeared $[b=1.08$, $t(2008)=1.51, p=0.132]$.

In Experiment 2, we found similar results as those found with children in Experiment 1. Males performed better than females in approximate arithmetic. Controlling for the mental

TABLE 5 | Means, standard deviations, and gender differences for all tasks (Experiment 2).

\begin{tabular}{|c|c|c|c|c|c|}
\hline \multirow[t]{2}{*}{ Tasks } & \multicolumn{2}{|c|}{ Arts } & \multicolumn{2}{|c|}{ Science } & \multirow{2}{*}{$\begin{array}{c}\text { Gender difference } \\
\text { F }\end{array}$} \\
\hline & Males & Females & Males & Females & \\
\hline Approximate arithmetic & $79.9(12.8)$ & $77.5(10.2)$ & $81.9(12.0)$ & $80.2(9.7)$ & $-2.11^{*}$ \\
\hline Mental rotation & $27.4(8.0)$ & $24.5(8.4)$ & $28.9(9.6)$ & $26.3(7.9)$ & $-3.71^{* * *}$ \\
\hline Word semantic processing & $36.2(8.6)$ & $40.6(6.4)$ & $36.0(6.5)$ & $38.3(6.3)$ & $4.95^{* * *}$ \\
\hline Spatial working memory & $149.5(27.5)$ & $153.8(18.7)$ & $154.1(22.6)$ & $154.5(20.2)$ & 1.16 \\
\hline Raven's Progressive Matrices & $23.1(7.4)$ & $23.3(7.0)$ & $22.8(6.6)$ & $23.8(5.7)$ & 0.94 \\
\hline
\end{tabular}

${ }^{*} p<0.05 ;{ }^{* * *} p<0.001$. 
TABLE 6 | Correlations among all tasks (Experiment 2).

\begin{tabular}{llcccc}
\hline Tasks & $\mathbf{1}$ & $\mathbf{2}$ & $\mathbf{3}$ & $\mathbf{4}$ \\
\hline 1 & Approximate arithmetic & - & & & \\
2 & Mental rotation & $0.17^{* * *}$ & - & & \\
3 & Word semantic processing & $0.10^{*}$ & $0.12^{* *}$ & - & \\
4 & Spatial working memory & $0.18^{* * *}$ & $0.22^{* * *}$ & $0.13^{* *}$ & - \\
5 & Raven's Progressive Matrices & $0.22^{* * *}$ & $0.25^{* * *}$ & $0.24^{* * *}$ & $0.26^{* * *}$ \\
\hline${ }^{*} p<0.05 ;{ }^{* *} p<0.01 ;{ }^{* * *} p<0.001$ & & & &
\end{tabular}

TABLE 7 | Results from multilevel modeling showing gender differences in approximate arithmetic (Experiment 2).

\begin{tabular}{lccc}
\hline \multirow{2}{*}{ Covariate } & \multicolumn{3}{c}{ Approximate arithmetic } \\
\cline { 2 - 4 } & $\boldsymbol{b}$ & SE $\times \boldsymbol{b}$ & $\boldsymbol{t}$ \\
\hline None & -2.01 & 0.95 & $t(551)=-2.11^{*}$ \\
Mental rotation & -1.50 & 0.96 & $t(550)=-1.57$ \\
Word semantic processing & -2.78 & 0.97 & $t(550)=-2.87^{* *}$ \\
Spatial working memory & -2.22 & 0.94 & $t(550)=-2.37^{*}$ \\
Raven's Progressive Matrices & -2.26 & 0.93 & $t(550)=-2.43^{*}$ \\
\hline$* p<0.05$ * * * $^{*}<0.01$. Gender was & &
\end{tabular}

rotation task, gender difference disappeared; but controlling for the other cognitive tasks, males still had an advantage over females.

\section{DISCUSSION}

The goal of the current study was to examine gender differences in approximate arithmetic. Our results showed that males performed better in approximate arithmetic than did females, and this gender difference disappeared after controlling for spatial ability.

\section{Cognitive Mechanism of Approximate Arithmetic}

Approximate arithmetic has a high correlation with spatial ability. Behavioral studies showed that participants represented numerical magnitude on the mental number line in the symbolic and non-symbolic approximate arithmetic tasks (McCrink and Wynn, 2004; Knops et al., 2009) and that the mental number line has a spatial property (Dehaene et al., 1993). Neuroimaging studies have shown that approximate arithmetic and spatial processing share a similar brain basis, typically involving the parietal cortex (Dehaene et al., 1999; Stanescu-Cosson et al., 2000; Lemer et al., 2003). Compared to the non-mathematician control group, mathematicians excelled in approximate arithmetic (Dowker, 1992; Dowker et al., 1996) and their parietal cortex (a brain region involved in spatial processing, (Corbetta et al., 1998; Kosslyn et al., 1998; Gitelman et al., 1999; Zacks et al., 1999) showed greater gray matter density (Aydin et al., 2007).

Approximate arithmetic relies on spatial ability, but not on language ability. In a study of language and approximate arithmetic (Spelke and Tsivkin, 2001), bilingual students were trained to perform exact and approximate arithmetic problems in two languages. Results showed that, for exact arithmetic, the language used for training mattered, but for approximate arithmetic, the language used for training did not matter. A recent study also found that children with language impairment had lower accuracy in exact arithmetic, but they had similar performance in approximate arithmetic as compared to the normal children (Nys et al., 2013). From a developmental perspective, approximate arithmetic precedes exact arithmetic because the latter relies on number symbols as language processing. For example, preschool children can perform approximate arithmetic but not exact arithmetic with the same numbers (Gilmore et al., 2007). Similarly, Amazonian indigenes can perform approximate arithmetic, but not exact arithmetic, due to their lack of a formal language-based number system (Pica et al., 2004).

\section{Gender Difference in Spatial Ability}

Many studies have shown that males outperform females on spatial ability tasks, especially the mental rotation tasks (Voyer et al., 1995). Gender difference in spatial ability emerges as early as about 3-5 months of age (Moore and Johnson, 2008; Quinn and Liben, 2008) and is evident to the age of 95 years (De Frias et al., 2006; Tran and Formann, 2008). Moreover, based on data from more than 200,000 subjects from 53 nations, Lippa et al. (2010) showed that males performed better than females on visuospatial tasks.

Neuroimaging studies have showed that males have a larger parietal lobule (Frederikse et al., 1999), which could explain males' superiority in spatial ability (Koscik et al., 2009). The right parietal cortex is involved in visuospatial processing during arithmetic tasks (see Arsalidou and Taylor, 2011, for a meta-analysis). For example, when the right parietal cortex was suppressed, participants could not perform spatial tasks (Bjoertomt et al., 2002; Rosenthal et al., 2009). Interestingly, when males perform the spatial tasks, their bilateral hemispheres are involved, whereas females tend to rely on their right hemisphere (Gur et al., 2000; Clements et al., 2006). Taken together, it is plausible that males' larger parietal cortex (especially in the right hemisphere, Caviness et al., 1996; Baibakov and Fedorov, 2010) accounts for their better performance on spatial tasks (Moore and Johnson, 2008; Quinn and Liben, 2008).

In sum, our study showed consistent gender differences in approximate arithmetic favoring males across age groups and identified gender differences in spatial ability as a potential cognitive mechanism. These results have important implications for later development of mathematical cognition. Future research should pay more attention to the understudied approximate arithmetic, which may be important for advanced mathematics.

\section{AUTHOR CONTRIBUTIONS}

WW: designed the experiment, collected and analyzed the data and drafted the manuscript; CC: revised the manuscript; XZ: designed the experiment, revised the manuscript. 


\section{ACKNOWLEDGMENTS}

This research was supported by the National Key Basic Research Program of China (No. 2014CB846100), by

\section{REFERENCES}

Arsalidou, M., and Taylor, M. J. (2011). Is $2+2=4$ ? Meta-analyses of brain areas needed for numbers and calculations. Neuroimage 54, 2382-2393. doi: 10.1016/j.neuroimage.2010.10.009

Aydin, K., Ucar, A., Oguz, K. K., Okur, O. O., Agayev, A., Unal, Z., et al. (2007). Increased gray matter density in the parietal cortex of mathematicians: a voxel-based morphometry study. Am. J. Neuroradiol. 28, 1859-1864. doi: 10.3174/ajnr.A0696

Baibakov, S. E., and Fedorov, V. P. (2010). Morphometric characteristics of the brain in children aged one year (magnetic resonance tomography data). Neurosci. Behav. Physiol. 40, 69-72. doi: 10.1007/s11055-009-9224-5

Bjoertomt, O., Cowey, A., and Walsh, V. (2002). Spatial neglect in near and far space investigated by repetitive transcranial magnetic stimulation. Brain 125, 2012-2022. doi: 10.1093/brain/awf211

Caviness, V. S. Jr., Kennedy, D. N., Richelme, C., Rademacher, J., and Filipek, P. A. (1996). The human brain age 7-11 years: a volumetric analysis based on magnetic resonance images. Cereb. Cortex 6, 726-736. doi: $10.1093 /$ cercor/6.5.726

Ceci, S. J., Williams, W. M., and Barnett, S. M. (2009). Women's underrepresentation in science: sociocultural and biological considerations. Psychol. Bull. 135:218. doi: 10.1037/a0014412

Clements, A. M., Rimrodt, S. L., Abel, J. R., Blankner, J. G., Mostofsky, S. H., Pekar, J. J., et al. (2006). Sex differences in cerebral laterality of language and visuospatial processing. Brain Lang. 98, 150-158. doi: 10.1016/j.bandl.2006.04.007

Corbetta, M., Akbudak, E., Conturo, T. E., Snyder, A. Z., Ollinger, J. M., Drury, H. A., et al. (1998). A common network of functional areas for attention and eye movements. Neuron 21, 761-773. doi: 10.1016/S0896-6273(00)80593-0

De Frias, C. M., Nilsson, L.-G., and Herlitz, A. (2006). Sex differences in cognition are stable over a 10-year period in adulthood and old age. Aging Neuropsychol. Cogn. 13, 574-587. doi: 10.1080/13825580600678418

Dehaene, S., Bossini, S., and Giraux, P. (1993). The mental representation of parity and number magnitude. J. Exp. Psychol. 122, 371-396. doi: 10.1037/00963445.122.3.371

Dehaene, S., Spelke, E., Pinel, P., Stanescu, R., and Tsivkin, S. (1999). Sources of mathematical thinking: behavioral and brain-imaging evidence. Science 284, 970-974. doi: 10.1126/science.284.5416.970

Dowker, A. (1992). Computational estimation strategies of professional mathematicicans. J. Res. Math. Educ. 23, 45-55. doi: 10.2307/749163

Dowker, A., Flood, A., Griffiths, H., Harriss, L., and Hook, L. (1996). Estimation strategies of four groups. Math. Cogn. 2, 113-135. doi: $10.1080 / 135467996387499$

Else-Quest, N. M., Hyde, J. S., and Linn, M. C. (2010). Cross-national patterns of gender differences in mathematics: a meta-analysis. Psychol. Bull. 136:103. doi: $10.1037 / \mathrm{a} 0018053$

Frederikse, M. E., Lu, A., Aylward, E., Barta, P., and Pearlson, G. (1999). Sex differences in the inferior parietal lobule. Cereb. Cortex 9, 896-901. doi: $10.1093 /$ cercor/9.8.896

Gilmore, C. K., McCarthy, S. E., and Spelke, E. S. (2007). Symbolic arithmetic knowledge without instruction. Nature 447, 589-591. doi: 10.1038/nature 05850

Gitelman, D. R., Nobre, A. C., Parrish, T. B., LaBar, K. S., Kim, Y. H., Meyer, J. R., et al. (1999). A large-scale distributed network for covert spatial attention Further anatomical delineation based on stringent behavioural and cognitive controls. Brain 122, 1093-1106. doi: 10.1093/brain/122.6.1093

Guilford, J. P. (1936). The determination of item difficulty when chance success is a factor. Psychometrika 1, 259-264. doi: 10.1007/BF02287877

Guiso, L., Ferdinando, M., Paola, S., and Zingales, L. (2008). Culture, gender, and math. Science 320, 1164-1165. doi: 10.1126/science. 1154094 three grants from the Natural Science Foundation of China (Nos. 31271187, 31221003 and 31500902) and by grant from Ministry of Education of the PRC (No. mjzxyb1412).

Gur, R. C., Alsop, D., Glahn, D., Petty, R., Swanson, C. L., Maldjian, J. A., et al. (2000). An fMRI study of sex differences in regional activation to a verbal and a spatial task. Brain Lang. 74, 157-170. doi: 10.1006/brln.200 0.2325

Halpern, D. F., Benbow, C. P., Geary, D. C., Gur, R. C., Hyde, J. S., and Gernsbacher, M. A. (2007). The science of sex differences in science and mathematics. Psychol. Sci. Public Interest 8, 1-51. doi: 10.1111/j.1529-1006.2007.00032.x

Hyde, J. S., Lindberg, S. M., Linn, M. C., Ellis, A. B., and Williams, C. C. (2008). Gender similarities characterize math performance. Science 321, 494-495. doi: $10.1126 /$ science. 1160364

Hyde, J. S., and Linn, M. C. (2006). Gender similarities in mathematics and science. Science 314, 599-600. doi: 10.1126/science.1132154

Knops, A., Viarouge, A., and Dehaene, S. (2009). Dynamic representations underlying symbolic and nonsymbolic calculation: evidence from the operational momentum effect. Attent. Percept. Psychophys. 71, 803-821. doi: 10.3758/APP.71.4.803

Koscik, T., O'Leary, D., Moser, D. J., Andreasen, N. C., and Nopoulos, P. (2009). Sex differences in parietal lobe morphology: relationship to mental rotation performance. Brain Cogn. 69, 451-459. doi: 10.1016/j.bandc.2008.09.004

Kosslyn, S. M., DiGirolamo, G. J., Thompson, W. L., and Alpert, N. M. (1998). Mental rotation of objects versus hands: neural mechanisms revealed by positron emission tomography. Psychophysiology 35, 151-161. doi: 10.1111/1469-8986.3520151

Kovas, Y. H., Claire, M. A., Petrill, S. A., and Plomin, R. (2007). Mathematical ability of 10-Year-old boys and girls genetic and environmental etiology of typical and low performance. J. Learn. Disabil. 40, 554-567. doi: 10.1177/00222194070400060601

Lemer, C. D., Stanislas, S. E., and Cohen, L. (2003). Approximate quantities and exact number words: dissociable systems. Neuropsychologia 41, 1942-1958. doi: 10.1016/S0028-3932(03)00123-4

Levine, D. R. (1982). Strategy use and estimation ability of college students. J. Res. Math. Educ. 13, 350-359. doi: 10.2307/749010

Linn, M. C., and Hyde, J. S. (1989). Gender, mathematics, and science. Educ. Res. 18, 17-27. doi: 10.3102/0013189X018008017

Lippa, R. A., Collaer, M. L., and Peters, M. (2010). Sex differences in mental rotation and line angle judgments are positively associated with gender equality and economic development across 53 nations. Arch. Sex. Behav. 39, 990-997. doi: 10.1007/s10508-008-9460-8

McCrink, K., and Wynn, K. (2004). Large-number addition and subtraction by 9-month-old infants. Psychol. Sci. 15, 776-781. doi: 10.1111/j.09567976.2004.00755.x

Moore, D. S., and Johnson, S. P. (2008). Mental rotation in human infants a sex difference. Psychol. Sci. 19, 1063-1066. doi: 10.1111/j.1467-9280.2008. 02200.x

Nosek, B. A., Smyth, F. L., Sriram, N., Lindner, N. M., Devos, T., Ayala, A., et al. (2009). National differences in gender-science stereotypes predict national sex differences in science and math achievement. Proc. Natl. Acad. Sci. U.S.A. 106, 10593-10597. doi: 10.1073/pnas.0809921106

Nys, J., Content, A., and Leybaert, J. (2013). Impact of language abilities on exact and approxiamte number skills development: evidence from children with specific language impairment. J. Speech Lang. Hear Res. 56, 956-970. doi: 10.1044/1092-4388(2012/10-0229)

Peugh, J. L., and Enders, C. K. (2005). Using the SPSS mixed procedure to fit crosssectional and longitudinal multilevel models. Educ. Psychol. Measur. 65:717. doi: 10.1177/0013164405278558

Pica, P., Lemer, C., Izard, V., and Dehaene, S. (2004). Exact and approximate arithmetic in an Amazonian indigene group. Science 306, 499-503. doi: 10.1126/science. 1102085

Quinn, P. C., and Liben, L. S. (2008). A sex difference in mental rotation in young infants. Psychol. Sci. 19, 1067-1070. doi: 10.1111/j.1467-9280.2008. 02201.x 
Raven, J. (1998). Manual for Raven's Progressive Matrices and Vocabulary Scales. Oxford: Oxford Psychologists Press.

Rosenthal, C. R., Roche-Kelly, E. E., Husain, M., and Kennard, C. (2009). Responsedependent contributions of human primary motor cortex and angular gyrus to manual and perceptual sequence learning. J. Neurosci. 29, 15115-15125. doi: 10.1523/JNEUROSCI.2603-09.2009

Rubenstein, R. N. (1985). Computational estimation and related mathematical skills. J. Res. Math. Educ. 16, 106-119. doi: 10.2307/748368

Shen, H. (2013). Inequality quantified: mind the gender gap. Nature 495, 22-24. doi: $10.1038 / 495022 a$

Shepard, R. N., and Metzler, J. (1971). Mental rotation of three-dimensional objects. Science 171, 701-703. doi: 10.1126/science.171.3972.701

Siegel, L. S., and Ryan, E. B. (1988). Development of grammatical-sensitivity, phonological, and short-term memory skills in normally achieving and learning disabled children. Dev. Psychol. 24, 28-37. doi: 10.1037/0012-1649.24.1.28

So, D., and Siegel, L. S. (1997). Learning to read Chinese: semantic, syntactic, phonological and working memory skills in normally achieving and poor Chinese readers. Read. Writ. 9, 1-21. doi: 10.1023/A:1007963513853

Spelke, E. S. (2005). Sex differences in intrinsic aptitude for mathematics and science? A critical review. Am. Psychol. Am. Psychol. 60, 950-958. doi: 10.1037/0003-066X.60.9.950

Spelke, E. S., and Tsivkin, S. (2001). Language and number: a bilingual training study. Cognition 78, 45-88. doi: 10.1016/S0010-0277(00)00108-6

Stanescu-Cosson, R., Pinel, P., van De Moortele, P. F., Le Bihan, D., Cohen, L., and Dehaene, S. (2000). Understanding dissociations in dyscalculia A brain imaging study of the impact of number size on the cerebral networks for exact and approximate calculation. Brain 123, 2240-2255. doi: 10.1093/brain/123. 11.2240

Tran, U. S., and Formann, A. K. (2008). Piaget's water-level tasks: performance across the lifespan with emphasis on the elderly. Pers. Individ. Differ. 45, 232-237. doi: 10.1016/j.paid.2008.04.004

Voyer, D., Voyer, S., and Bryden, M. P. (1995). Magnitude of sex differences in spatial abilities: a meta-analysis and consideration of critical variables. Psychol. Bull. 117, 250-270. doi: 10.1037/0033-2909.117.2.250

Wei, W., Lu, H., Zhao, H., Chen, C., Dong, Q., and Zhou, X. (2012). Gender differences in children's arithmetic performance are accounted for by gender differences in language abilities. Psychol. Sci. 23, 320-330. doi: 10.1177/0956797611427168

Zacks, J., Rypma, B., Gabrieli, J. D., Tversky, B., and Glover, G. H. (1999). Imagined transformations of bodies: an fMRI investigation. Neuropsychologia 37, 1029-1040. doi: 10.1016/S0028-3932(99)00012-3

Conflict of Interest Statement: The authors declare that the research was conducted in the absence of any commercial or financial relationships that could be construed as a potential conflict of interest.

Copyright (c) 2016 Wei, Chen and Zhou. This is an open-access article distributed under the terms of the Creative Commons Attribution License (CC BY). The use, distribution or reproduction in other forums is permitted, provided the original author(s) or licensor are credited and that the original publication in this journal is cited, in accordance with accepted academic practice. No use, distribution or reproduction is permitted which does not comply with these terms. 